\section{Band 28, Heft 2, April 2012}

Editorial

84 Das Karzinom am ösophagogastralen Übergang Hölscher, A.H. (Köln); Malfertheiner, P. (Magdeburg)

Hauptthema

86 Das Karzinom am ösophagogastralen Übergang: Epidemiologische Trends und Risikofaktoren

Bornschein, J.; Selgrad, M.; Malfertheiner, P. (Magdeburg)

96 Molekulare Grundlagen und Pathomechanismen des Karzinoms am ösophagogastralen Übergang

Selgrad, M.; Bornschein, J.; Weigt, J.; Malfertheiner, P. (Magdeburg)

103 Barrett-Ösophagus und -Neoplasie am ösophagogastralen Übergang: Ein Leitfaden für die Routine

Veits, L.; Drgac, J. (Bayreuth); Neumann, H. (Erlangen);

Langner, C. (Graz); Vieth, M. (Bayreuth)

113 Multimodale Therapie mit kurativem Ansatz Hölscher, A.H.; Fetzner, U.K. (Köln)

121 Palliative und supportive Therapie beim Karzinom des ösophagogastralen Übergangs Block, A.; Arnold, D. (Hamburg)

129 Neue S3-Leitlinie zur Diagnostik und Therapie der Adenokarzinome des Magens und des ösophagogastralen Übergangs

Andus, T. (Stuttgart); Palmqvist, A. (Mainz);

Arends, J. (Freiburg i.Br.); Möhler, M. (Mainz)

Interdisziplinäres Gespräch

133 Das Karzinom am ösophagogastralen Übergang Günther, T.; Lippert, H. (Magdeburg) (Gesprächsleiter)

Originalarbeit

138 Komplikationsmanagement in der Narbenhernienchirurgie: Erhalt von alloplastischem Material in der Infektsituation

Philipp, M.; Förster, S.; Klar, E. (Rostock)
Vol. 28, Issue 2, April 2012

Editorial

84 Adenocarcinoma at the Esophagogastric Junction Hölscher, A.H. (Köln); Malfertheiner, P. (Magdeburg)

Main Topic

86 Adenocarcinoma at the Esophagogastric Junction: Epidemiological Trends and Risk Factors

Bornschein, J.; Selgrad, M.; Malfertheiner, P. (Magdeburg)

96 Molecular Background and Pathogenesis of the Adenocarcinoma at the Oesophagogastric Junction Selgrad, M.; Bornschein, J.; Weigt, J.; Malfertheiner, P. (Magdeburg)

103 Barrett's Esophagus and Neoplasia at the Esophagogastric Junction: Diagnostic Guidelines Veits, L.; Drgac, J. (Bayreuth); Neumann, H. (Erlangen); Langner, C. (Graz); Vieth, M. (Bayreuth)

113 Multimodal Therapy with Curative Approach Hölscher, A.H.; Fetzner, U.K. (Köln)

121 Multimodal Treatment and Supportive Care of Advanced Adenocarcinoma of the Esophagogastric Junction Block, A.; Arnold, D. (Hamburg)

129 New German S3-Guideline 'Diagnosis and Treatment of Esophagogastric Cancer' Andus, T. (Stuttgart); Palmqvist, A. (Mainz); Arends, J. (Freiburg i.Br.); Möhler, M. (Mainz)

Interdisciplinary Discussion

133 Adenocarcinoma at the Esophagogastric Junction Günther, T.; Lippert, H. (Magdeburg) (Discussion Leader)

Original Article

138 Complicational Management Following Surgery of Incisional Hernia: Preservation of Alloplastic Material in the Case of Infection Philipp, M.; Förster, S.; Klar, E. (Rostock)

\section{KARGER}

Fax +497614520714

Information@Karger.de

www.karger.com (c) 2012 S. Karger GmbH, Freiburg 


\section{Band 28, Heft 2, April 2012}

Klinische Information

142 Aszites - Diagnostik, Therapie und Sekundärkomplikationen

Sebode, M.; Benten, D. (Hamburg)

149 News / Ticker

152 Tagungen und Kongresse

148 Impressum

156 Hinweise für die Autoren
Vol. 28, Issue 2, April 2012

Clinical Information

142 Ascites - Clinical Diagnostics, Treatment and Secondary Complications

Sebode, M.; Benten, D. (Hamburg)

149 News / Ticker

152 Meetings and Conferences

148 Imprint

156 Guidelines for Authors

\section{KARGER}

Fax +497614520714 (c) 2012 S. Karger GmbH, Freiburg

Accessible online at: www.karger.com/vim 